\title{
Services d'injection supervisée : mesure d'intervention communautaire en réponse à la crise des opioïdes à Ottawa (Canada)
}

Sarah DelVillano, B.A. (1); Margaret de Groh, Ph. D. (2); Howard Morrison, Ph. D. (2); Minh T. Do, Ph. D. $(3,4,5)$

Diffuser cet article sur Twitter

\section{Résumé}

L'établissement de services d'injection supervisée (SIS) est devenu courant dans les collectivités à risque élevé afin de réagir à la crise des opioïdes qui sévit actuellement au Canada. Le service de La Roulotte (The Trailer), qui a ouvert ses portes en novembre 2017 à Ottawa (Canada), suit de près la consommation des clients, le traitement des surdoses et l'inversion des effets d'une surdose. Nous avons analysé les données recueillies entre novembre 2017 et août 2018 par ce service. Aux heures de pointe, la demande de services a constamment dépassé la capacité de La Roulotte. Le nombre de traitements de surdoses et d'inversions des effets d'une surdose a considérablement augmenté au cours de la période. D’après les résultats, La Roulotte a fourni un service important - quoique non optimal (en raison de contraintes d'espace) - de réduction des méfaits dans cette collectivité à risque élevé.

Mots-clés : services d'injection supervisée, centres d'injection supervisée, salles de consommation à moindre risque, réduction des méfaits, dépendance, opioïdes, naloxone

\section{Introduction}

Le Canada traverse actuellement une crise des opioïdes ${ }^{1}$. Le nombre de décès apparemment liés aux opioïdes au Canada est passé de 2978 en 2016 à 3987 en 2017, soit une augmentation de $34 \%^{2}$. Cette hausse est en grande partie attribuable à une toxicité croissante dans l'approvisionnement en drogues illicites ${ }^{2,3}$. Les services d'injection supervisée (SIS) constituent un modèle de réduction des méfaits destiné à atténuer les effets de l'usage d'opioïdes.

D'après de récentes revues systématiques ${ }^{4,5}$, les SIS implantés dans les collectivités permettent de réduire la mortalité et la morbidité par surdose chez les personnes qui y ont recours, d'accroître les comportements de réduction des méfaits (diminution du partage et de la réutilisation des seringues) et d'augmenter l'entame de services de traitement des troubles liés à la consommation de substances. Le modèle des SIS s'est également révélé rentable, et il contribue à réduire la pression sur les services locaux, en particulier les services médicaux d'urgence (SMU) ${ }^{4,6}$. L'établissement et le fonctionnement des SIS dans les collectivités au Canada demeure cependant un modèle de réduction des méfaits controversé ${ }^{7,8,9}$.

En novembre 2017, à la suite d'une augmentation très rapide du nombre de décès liés à l'injection d'opioïdes dans la ville d'Ottawa (Canada), a été créé le service d’injection supervisée " La Roulotte », géré par l'organisme Ottawa Inner City Health Inc. (OICHI) avec l'autorisation du gouvernement du Canada. La Roulotte est située

\section{Points saillants}

- La Roulotte (service d'injection supervisée) a été établie en réponse à la crise des opioïdes à Ottawa, Canada.

- La Roulotte offre un service 24 heures sur 24 aux clients.

- Le nombre d'inversions des effets d'une surdose effectuées a augmenté considérablement au cours de la période de suivi.

- La demande de services a constamment dépassé la capacité de La Roulotte.

dans un secteur d'Ottawa où la consommation de drogues est élevée, à proximité de services d'hébergement et de traitement pour personnes consommatrices de drogue dans la collectivité. Cet aperçu offre un bilan sur les clients qui fréquentent La Roulotte et sur les traitements des surdoses qui y sont dispensés. La plupart des résultats sont fondés sur le rapport annuel présenté par La Roulotte à Santé Canada ${ }^{10}$.

\section{Méthodologie}

Le personnel de La Roulotte est chargé de faire un suivi des services et de présenter des résultats trimestriels au gouvernement provincial de l'Ontario, ainsi qu'un rapport annuel à Santé Canada. La plupart des résultats que nous présentons ici sont fondés sur le suivi effectué entre novembre 2017 et août 2018 (les résultats d'août 2018

Rattachement des auteurs :

1. Ottawa Inner City Health Inc., Ottawa (Ontario), Canada

2. Agence de la santé publique du Canada, Ottawa (Ontario), Canada

3. Santé Canada, Ottawa (Ontario), Canada

4. École de santé publique Dalla Lana, Université de Toronto, Toronto (Ontario), Canada

5. Département des sciences de la santé, Université Carleton, Ottawa (Ontario), Canada

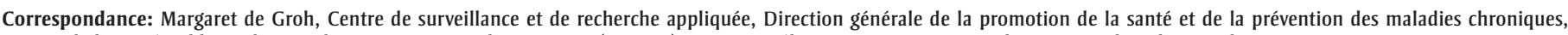
Agence de la santé publique du Canada, 785, avenue Carling, Ottawa (Ontario) K1S 5H4; tél. : 613 614-2045; courriel : margaret.degroh@canada.ca 
ne sont pas disponibles dans le rapport annuel).

Les clients de La Roulotte collaborent avec le personnel afin d'établir un identificateur unique, facile à mémoriser, qui est destiné à préserver leur anonymat tout en permettant de faire un suivi de leur fréquentation du SIS.

La Roulotte fait un suivi de plusieurs marqueurs de données clés, en particulier les visites individuelles, les types de drogues utilisées (signalées par le client seulement), le nombre d'injections effectuées, le nombre de surdoses et les problèmes de santé observables (p. ex. abcès, plaies).

Pour les surdoses, le type de traitement appliqué fait l'objet d'un suivi. Dans le cas des clients qui présentent des signes de surdose et qui respirent, l'oxygénothérapie (" administration d'oxygène »), la respiration de sauvetage et la stimulation sont utilisées. Dans les cas où l'on procède uniquement à une administration d'oxygène, le traitement de la surdose est alors classé dans cette catégorie. Si le client ne respire pas ou ne répond pas à l'administration d'oxygène ou à la stimulation, on procède à l'administration de naloxone («administration de naloxone»).

Selon l'expérience du personnel de La Roulotte, de nombreux clients se font plusieurs injections (2 ou 3) au cours d'une même visite. Bien que ce soit le nombre d'injections qui fasse l'objet du suivi, nous avons établi le taux de surdose pour 1000 visites plutôt que pour 1000 injections, comme c'est le cas habituellement, étant donné que la plupart des cas de surdose étaient jusqu'à présent liés à des injections uniques ${ }^{11,12}$. Une telle approche tient compte de l'évolution des méthodes d'injection de la drogue au fil du temps, et par conséquent donne une meilleure indication des cas de surdoses.

\section{Résultats}

\section{Profil de la clientèle}

Au 19 octobre 2018, 1049 clients s'étaient inscrits à La Roulotte depuis sa création en novembre 2017 (communication personnelle de l'OICHI, 2018). Ce nombre exclut les clients qui sont décédés depuis novembre 2017 (six décès connus). Entre novembre 2017 et août 2018, La Roulotte a fourni des services à en moyenne 226 clients différents chaque mois ${ }^{10}$. L'OCIHI indique que sa clientèle est demeurée relativement stable et que le taux de roulement mensuel avoisinait les $13 \%$.

Près des trois quarts des clients se sont identifiés comme étant des hommes et la majorité (60\%) avait entre 25 et 45 ans. Douze pour cent des clients ont déclaré avoir entre 18 et 25 ans.

Les produits opiacés ont été, de loin, le plus souvent cités comme drogues injectées à La Roulotte (figure 1), bien que leur consommation déclarée ait chuté entre le début et la fin de la période de suivi, passant d'environ $75 \%$ de toutes les injections à un peu plus de $50 \%$. Le pourcentage de personnes ayant déclaré s'être injecté de la cocaïne ou de la cocaïne épurée (crack) a augmenté légèrement au fil du temps.

\section{Recours à des services d'injection supervisée}

La Roulotte offre un service 24 heures sur 24 aux clients. Le tableau 1 présente le nombre mensuel total de visites individuelles à La Roulotte et le nombre quotidien moyen de visites entre novembre 2017 et août 2018. Alors qu'on s'attendait, au départ, à ce que La Roulotte accueille de 60 à 80 visites par jour, le service reçoit en moyenne 121 visites par jour. C'est en fin d'après-midi et en soirée que la demande est la plus élevée, et elle excède presque toujours la disponibilité des cabines d'injection. Aux heures de pointe, les clients doivent, de ce fait, attendre à l'extérieur que des cabines se libèrent.
Le tableau 1 indique également le nombre mensuel de traitements des surdoses (administration d'oxygène ou de naloxone) à l'intérieur de l'établissement. Le nombre de surdoses ayant donné lieu à un appel aux SMU (soit entre 0 et 2 par mois) n'est pas compris dans ces estimations.

Le type de traitement appliqué est demeuré pratiquement le même chaque mois, avec une moyenne mensuelle globale de 29 administrations d'oxygène et de 28 administrations de naloxone. La figure 2 présente le taux mensuel d'inversion des effets d'une surdose par tranche de 1000 visites à La Roulotte, par administration de naloxone, par administration d'oxygène et pour les deux traitements combinés. Toutes les tendances sont statistiquement significatives. Pour l'administration de naloxone uniquement, la tendance linéaire statistiquement significative $(\mathrm{p}<0,05)$ indique que le nombre mensuel d'inversions des effets d'une surdose a augmenté de 5 au cours de la période visée. L'OICHI signale (communication personnelle, 2018) que le nombre d'inversions des effets d'une surdose effectuées pendant la période de suivi (administration de naloxone seulement) correspond à 112 clients différents.

\section{Analyse}

La crise des opioïdes, alimentée par un approvisionnement en drogues illicites toxiques imprévisible et sans précédent, a entraîné un besoin urgent de services d'injection supervisée. L'emplacement d'un SIS, l'accessibilité à celui-ci et l'attitude du

FIGURE 1

Consommation mensuelle déclarée de drogues injectables à La Roulotte, entre novembre 2017 et août 2018, Ottawa (Ontario), Canada

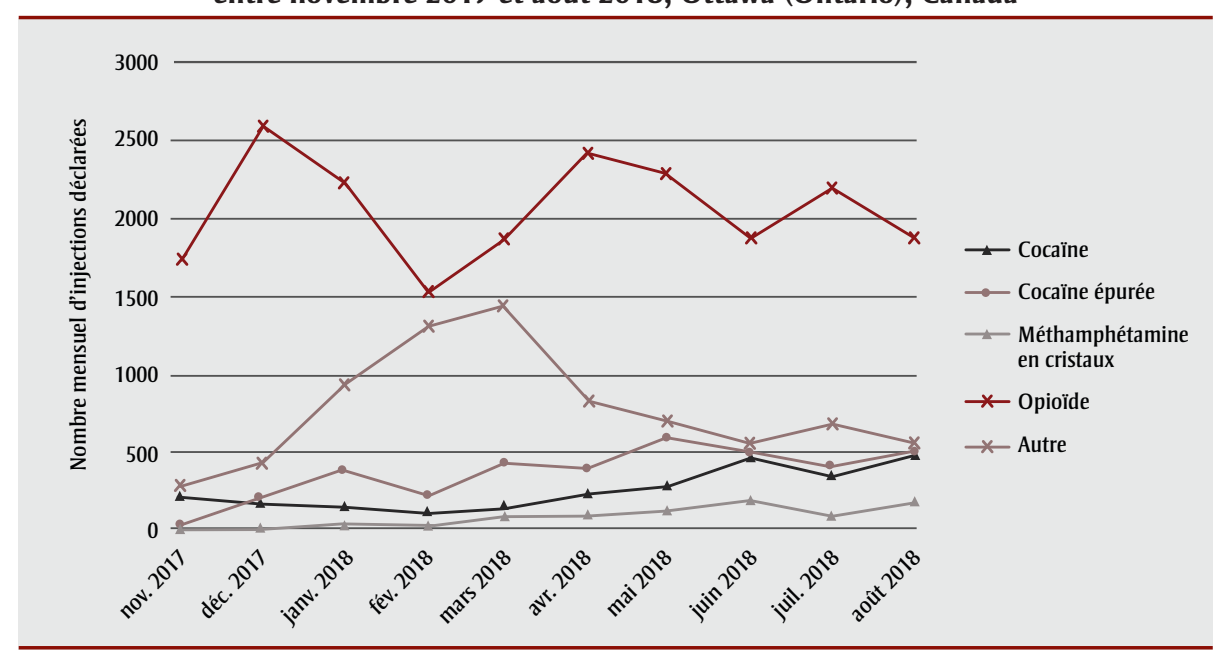

Source des données : Ottawa Inner City Health Inc. (OICHI), Report to Health Can SIS, November 2017-September 2018 ${ }^{10}$. 
TABLEAU 1

Nombre de visites à La Roulotte et nombre de traitements de surdoses par mois, entre novembre 2017 et août 2018, Ottawa (Ontario), Canada

\begin{tabular}{|c|c|c|c|c|c|c|c|c|c|c|}
\hline $\begin{array}{l}\text { Visites ou } \\
\text { traitements }\end{array}$ & $\begin{array}{l}\text { Nov. } \\
2017\end{array}$ & $\begin{array}{l}\text { Déc. } \\
2017\end{array}$ & $\begin{array}{l}\text { Janv. } \\
2018\end{array}$ & $\begin{array}{l}\text { Févr. } \\
2018\end{array}$ & $\begin{array}{l}\text { Mars } \\
2018\end{array}$ & $\begin{array}{l}\text { Avril } \\
2018\end{array}$ & $\begin{array}{c}\text { Mai } \\
2018\end{array}$ & $\begin{array}{l}\text { Juin } \\
2018\end{array}$ & $\begin{array}{c}\text { Juillet } \\
2018\end{array}$ & $\begin{array}{l}\text { Août } \\
2018\end{array}$ \\
\hline $\begin{array}{l}\text { Nombre mensuel de } \\
\text { visites }\end{array}$ & 3061 & 3171 & 4070 & 3435 & 3621 & 4129 & 3146 & 3626 & 3730 & 4192 \\
\hline $\begin{array}{l}\text { Nombre quotidien } \\
\text { moyen de visites }\end{array}$ & $127,5^{a}$ & 102,3 & 131,3 & 122,8 & 116,8 & 138,0 & 101,5 & 120,9 & 120,3 & 135,2 \\
\hline $\begin{array}{l}\text { Nombre de surdoses } \\
\text { traitées par } \\
\text { administration } \\
\text { d'oxygène seulement }\end{array}$ & 13 & 24 & 15 & 10 & 16 & 9 & 35 & 36 & 35 & 90 \\
\hline $\begin{array}{l}\text { Nombre de surdoses } \\
\text { dont les effets ont } \\
\text { été inversés par } \\
\text { administration de } \\
\text { naloxone }\end{array}$ & 15 & 24 & 16 & 11 & 20 & 14 & 39 & 40 & 36 & 79 \\
\hline
\end{tabular}

Source des données : Ottawa Inner City Health Inc. (OICHI), Report to Health Can SIS, November 2017-September $2018^{10}$. a Sur une période de 24 jours, La Roulotte ayant ouvert ses portes le 7 novembre 2017.

personnel envers les clients sont susceptibles de jouer un rôle essentiel dans son succès ou son échec ${ }^{7,13}$. La Roulotte a été en mesure de répondre aux besoins de nombreux clients parce qu'elle est située dans le secteur qu'elle dessert, qu'elle est ouverte tous les jours 24 heures sur 24 et qu'elle adopte un modèle qui procure un espace sécuritaire, exempt de honte pour ses clients, ce qui inspire la confiance nécessaire pour mettre en place les services de santé et de toxicomanie dont ils ont besoin $^{4,13,14}$. Toutefois, comme les besoins en services de La Roulotte durant les heures de pointe ont dépassé constamment sa capacité, les clients trouvant l'attente difficile ont risqué davantage de se blesser ou de décéder en s'injectant leur drogue sans

FIGURE 2

Taux d'inversion des effets d'une surdose par administration d'oxygène ou de naloxone, entre novembre 2017 et août 2018, Ottawa (Ontario), Canada

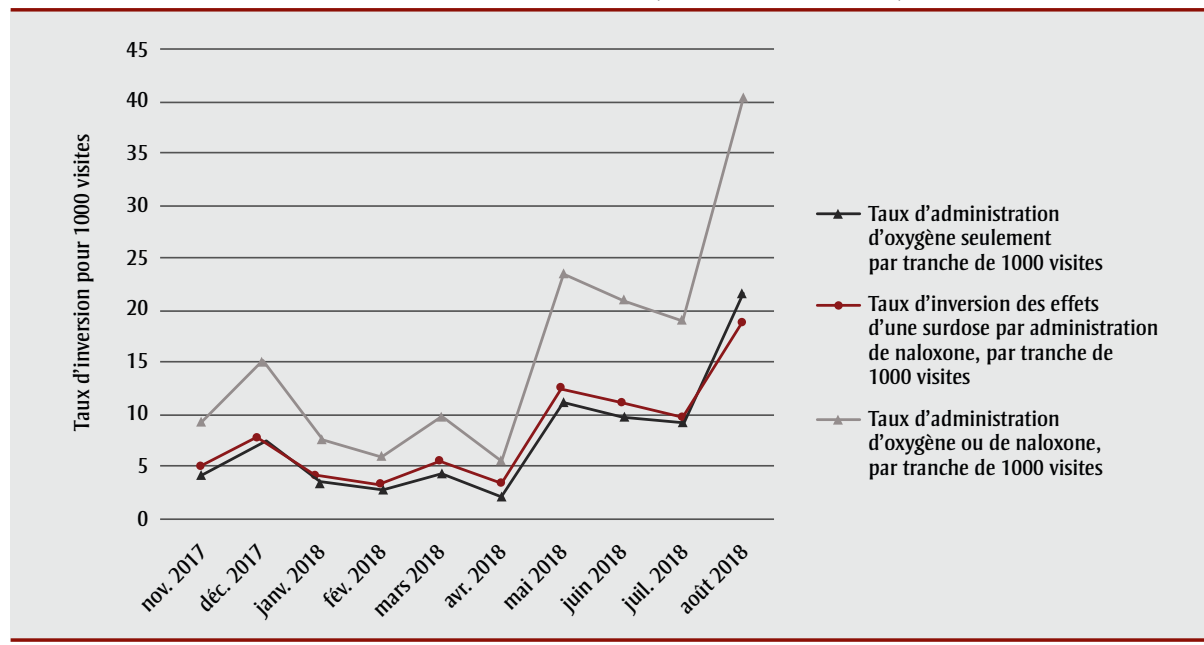

Source des données : Ottawa Inner City Health Inc. (OICHI), Report to Health Can SIS, November 2017-September $2018^{10}$. Remarque : L'administration de naloxone peut inclure une administration préalable d'oxygène qui n'a pas suffi à traiter la surdose. signaler l'introduction, dans un secteur, d'un " lot toxique ou de mauvaise qualité » de drogues illicites. Ainsi, c'est une augmentation rapide du nombre d'inversions des effets d'une surdose qui éveille l'attention de la communauté plutôt qu'une hausse rapide du nombre de décès par surdose.

La toxicité croissante des drogues illicites provoquée par l'introduction d'analogues du fentanyl en constante évolution ${ }^{3,15}$ rend difficile la prestation des services offerts par La Roulotte. Le personnel de La Roulotte a notamment dû faire face à une augmentation du nombre de surdoses traitées. En outre, le spectromètre situé au Centre communautaire de la Côte-de-Sable d'Ottawa a révélé la présence d'opioïdes synthétiques dans des drogues qui ne sont pas des opiacés, comme la cocaine épurée et la méthamphétamine ${ }^{3}$. Par conséquent, l'OICHI tente maintenant d'étendre ses services afin d'offrir un espace sûr aux personnes qui consomment des drogues par inhalation (« services de consommation supervisée $)^{10}$.

\section{Points forts et limites}

L'engagement du personnel de La Roulotte à préserver l'anonymat de ses clients rend la description détaillée de cette population très difficile. L'installation de La Roulotte dans un secteur où la consommation de drogues est élevée invite tout de même à penser que les clients ressemblent probablement à ceux qui ont fréquenté un SIS ayant du succès dans le passé (utilisateurs à long terme, clients marginalisés, personnes ayant peu de contacts avec le système de soins de santé) ${ }^{16,17}$. Il n'a pas été possible non plus d'évaluer la composition réelle des drogues utilisées, leur effet sur les cas de surdose ou les tendances en matière de surdose.

Nous avons été en mesure de fournir des estimations des cas de surdose en choisissant d'établir le taux de surdose par tranche de 1000 visites. À notre avis, étant données les changements dans les modèles d'injection de drogues, cette approche offre une estimation davantage comparable aux estimations habituelles des cas de surdose par le passé, particulièrement en ce qui concerne les surdoses d'hérö̈ne, qui surviennent habituellement après une seule injection $^{11,12}$. Bien que la définition officielle d'une "inversion des effets d'une surdose " corresponde à l'administration réussie de naloxone, nous avons également signalé les cas associés à l'administration 
d'oxygène. Il n'est pas possible de distinguer, parmi ces cas, ceux qui, sans administration d'oxygène, auraient entraîné une surdose nécessitant l'administration de naloxone.

Enfin, nous avons été incapables de déterminer si les clients fréquentant La Roulotte y font toutes leurs injections. Des études antérieures ont démontré que la fréquentation d'un SIS est loin d'atteindre les $100 \%{ }^{16,18}$. C'est probablement le cas des clients de La Roulotte, qui s'exposent ainsi à un risque lorsqu'ils doivent attendre qu'une cabine d'injection se libère ou lorsqu'ils décident de s'injecter la drogue dans des situations qui impliquent un risque élevé.

\section{Conclusion}

Le nombre de surdoses d'opioïdes évitées ou dont les effets ont été inversés par l'administration d'oxygène ou de naloxone a beaucoup augmenté au cours de la courte période écoulée depuis l'établissement de La Roulotte. La Roulotte n'a cependant pas été en mesure de répondre pleinement aux besoins des clients de la collectivité au cours de cette période ${ }^{10}$. Il reste à voir si la nouvelle Roulotte temporaire pourra répondre à la demande, avec ses quatre cabines additionnelles.

\section{Remerciements}

Nous tenons à souligner la contribution de XiaoHong Jiang à l'organisation des références de cet article. Nous tenons également à souligner le soutien de l'organisme Ottawa Inner City Health Inc. (OICHI) et de Wendy Muckle, notamment pour avoir fourni des renseignements complémentaires pour la rédaction de cet article et pour avoir autorisé la publication des résultats de l'OICHI.

\section{Conflits d'intérêts}

La première auteure (Sarah DelVillano) est pair aidant à La Roulotte. La deuxième auteure (MdG) était la rédactrice en chef par intérim au moment de la préparation de ce numéro de la Revue PSPMC, mais elle s'est soustraite à toutes les décisions éditoriales concernant ce manuscrit.

\section{Contributions des auteurs et avis}

SDV, MdG et MTD ont conçu et exposé les grandes lignes de l'article et ont mené les analyses. SDV, MdG et HM ont contribué à la rédaction de l'article. Tous les auteurs ont examiné de façon critique l'article et y ont apporté des révisions.

Approbation éthique : les analyses sont fondées sur des données agrégées accessibles au public et, à ce titre, l'approbation du Comité d'éthique et de la recherche n'a pas été nécessaire.

Le contenu de cet article et les points de vue qui y sont exprimés sont ceux des auteurs et ne reflètent pas nécessairement ceux du gouvernement du Canada.

\section{Références}

1. Orpana HM, Lang JJ, Baxi M, et al. Tendances canadiennes en matière de mortalité liée aux opioïdes et d'incapacité découlant d'un trouble de consommation d'opioïdes, à la lumière de l'Étude sur la charge mondiale de morbidité (1990-2014). Promotion de la santé et prévention des maladies chroniques au Canada. 2018;38(6): 267-277.

2. Comité consultatif spécial sur l'épidémie de surdoses d'opioïdes. Rapport national : décès apparemment liés à la consommation d'opioïdes au Canada [de janvier 2016 à décembre 2017] [Internet]. Ottawa (Ontario) : Agence de la santé publique du Canada; 2018. En ligne à : https://www.canada.ca/fr /sante-publique/services/publications /vie-saine/rapport-national-deces -apparemment-lies-consommation -opioides-publie-juin-2018.html

3. Centre de santé communautaire Côtede-Sable. Dépistage des drogues - Résultats [Internet]. Ottawa (Ont.) : Centre de santé communautaire Côte-de-Sable; 2018 [consultation le 26 octobre 2018]. En ligne à : https://www.shchc.ca/fr /programs/oasis/drug-checking

4. Potier C, Laprevote V, Dubois-Arber F, et al. Supervised injection services: what has been demonstrated? A systematic literature review. Drug Alcohol Depend. 2014;145:48-68.

5. Kennedy MC, Karamouzian M, Kerr T. Public health and public order outcomes associated with supervised drug consumption facilities: a systematic review. Curr HIV/AIDS Rep. 2017;14: 161-183.
6. Madah-Amiri D, Skulberg AK, Braarud $\mathrm{AC}$, et al. Ambulance-attended opioid overdoses: an examination into overdose locations and the role of a safe injection facility. Substance Abuse. 2018:1-6. doi: 10.1080/08897077.2018 .1485130 .

7. Kerr T, Mitra S, Kennedy MC, McNeil R. Supervised injection facilities in Canada: past, present, and future. Harm Reduct J. 2017;14(1):28.

8. Thomson E, Lampkin H, Maynard R, et al. The lessons learned from the fentanyl overdose crises in British Columbia, Canada. Addiction. 2017; 112:2068-2069.

9. Bayoumi A, Strike C. Making the case for supervised injection services. The Lancet Commentary. 2016;387:1890-1891.

10. Ottawa Inner City Health Inc (OICHI). Report to Health Can SIS [Internet]. Ottawa (Ont.) : OICHI; 2018 [consultation le 1er octobre 2018]. En ligne à : http://www.ottawainnercityhealth.ca /report-to-health-can-sis/

11. Roxburgh A, Darke S, Salmon AM, et al. Frequency and severity of non-fatal opioid overdoses among clients attending the Sydney Medically Supervised injection Centre. Drug and Alcohol Dependence. 2017;176:126-132.

12. Kerr T, Tyndall MW, Lai C, et al. Drugrelated overdoses within a medically supervised safer injection facility. Int J Drug Policy. 2006;17:436-441.

13. Collier R. Harm reduction is about providing safety for patients. CMAJ. 2017;189:E1154. doi: 10.1503/cmaj .1095489 .

14. McNeil R, Small W. "Safer environment interventions": a qualitative synthesis of the experiences and perceptions of people who inject drugs. Soc Sci Med. 2014;106:151-158.

15. Ontario HIV \& Substance Use Training Program. Naloxone resistant fentanyl caution needed about reports [Internet]. Toronto (Ont.) : Ontario HIV \& Substance Use Training Program; 2018. En ligne à : http://hklndrugstrategy.ca /wp-content/uploads/Naloxone-resistant -fentanyl-OHSUTP-Oct-2018.pdf 
16. Santé Canada. INSITE de Vancouver et autres sites d'injection supervisés : Observations tirées de la recherche Rapport final du Comité consultatif d'experts sur la recherche sur les sites d'injection supervisés. Ottawa (Ont.) : Santé Canada; 2008. En ligne à : https://www.canada.ca/fr/sante-canada /organisation/a-propos-sante-canada /rapports-publications/insite-vancouver -autres-sites-injection-supervises -observations-tirees-recherche.html

17. KPMG. NSW Health: Further evaluation of the Medically Supervised Injecting Centre during its extended Trial period (2007-2011). Final report. Australia : KPMG; 2010. En ligne à : https://www.health.nsw.gov.au/aod /resources/Documents/msic-kpmg.pdf

18. Hadland SE, DeBeck K, Kerr T, et al. Use of a medically supervised injection facility among street youth. Journal of Adolescent Health. 2014; 55:684-689. 